\title{
Small-angle neutron scattering studies of polybutadiene/polystyrene blends as a function of pressure and microstructure: Comparison of experiment and theory
}

\author{
Henrich Frielinghaus ${ }^{\mathrm{a})}$ and Dietmar Schwahn \\ Institut für Festkörperforschung, Forschungszentrum Jülich, D-52425 Jülich, Germany \\ Jacek Dudowicz, Karl F. Freed, and K. W. Foreman \\ The James Franck Institute and the Department of Chemistry, University of Chicago, Chicago, \\ Illinois 60637
}

(Received 15 November 2000; accepted 29 December 2000)

\begin{abstract}
Small-angle neutron scattering (SANS) experiments have been performed for three polybutadiene/ polystyrene $(\mathrm{dPB} / \mathrm{PS})$ blends of differing $\mathrm{dPB}$ microstructure as a function of pressure and temperature. The experimental effective SANS interaction parameters are analyzed using the mean-field lattice cluster theory (LCT). In order to provide a meaningful comparison with the LCT, contributions from the non-mean-field long-range composition fluctuations are removed from the experimental data by use of a crossover function that describes the transition between near-critical and mean-field behaviors for the extrapolated zero-angle scattering. The theory provides a good description of the overall pressure dependence of the effective interaction parameter and its small dependence on the percentage of 1,2 addition units in the dPB chains. (c) 2001 American Institute of Physics. [DOI: 10.1063/1.1350443]
\end{abstract}

\section{INTRODUCTION}

Apart from its relevance to understanding the behavior of polymers during processing, during certain high-pressure polymerizations, and under extreme conditions of usage, the study of the pressure dependence of polymer blend thermodynamic properties provides important insights into the molecular factors affecting the blend thermodynamics ${ }^{1,2}$ and thus the blend phase diagrams. These insights may be extracted from pressure-dependent experimental data by analyzing the data only with theories that treat the pressure dependence of the thermodynamic properties in a more general fashion than produced by the mere phenomenology of allowing the effective interaction parameter to vary with pressure.

The present paper describes small-angle neutron scattering (SANS) experiments for several blends of polybutadiene (dPB) and polystyrene (PS) that have been investigated as a function of pressure, temperature, and vinyl content. ${ }^{3} \mathrm{Be}-$ cause much of the SANS data is obtained for a temperature range that is not sufficiently far from the critical regime so that a mean-field description is valid, the SANS data are first analyzed with a crossover function ${ }^{4,5}$ that describes the transition between near-critical and mean-field behavior. This crossover analysis enables extraction of the mean-field susceptibility, which now may be compared meaningfully with mean-field theories. The experimental data are compared with the lattice cluster theory, ${ }^{6}$ which is able to predict the dependence of the SANS susceptibility on pressure, temperature, composition, molecular weights, and monomer molecular structures. More explicitly, the SANS experiments ${ }^{7}$ are interpreted in accordance with customary procedures by de-

${ }^{a)}$ Electronic mail: h.frielinghaus@fz-juelich.de termining the phenomenological effective Flory-Huggins (FH) parameter $\Gamma=-\Gamma_{\sigma}+\Gamma_{h} / T$, a segmental interaction parameter with the commonly observed temperature dependence that defines both enthalpic and entropic contributions. ${ }^{8}$ Studies of the pressure dependence of this interaction parameter ${ }^{9}$ serve to clarify the microscopic features affecting these individual contributions and their pressure dependence. For instance, the qualitative picture of "polymer packing" explains the observed decrease with pressure of the entropic contribution to the FH parameter because the polymers become more densely packed (i.e, have reduced excess free volume) at elevated pressures.

The lattice cluster theory of Freed and co-workers ${ }^{6}$ is a refined mean-field theory that includes contributions from nonrandom mixing and from the differing sizes and geometrical shapes of individual monomers. The lattice cluster theory (LCT) treats the pressure dependence of blend properties by using vacant lattice sites to represent the presence of excess free volume in compressible polymer systems in the liquid state. By describing the monomers with molecular structures extending over several lattice sites, ${ }^{10}$ the lattice cluster theory derives additional entropic and more-detailed energetic contributions to both the free-energy and the effective FH (SANS) interaction parameter. These novel features influence the predicted miscibility of polymer blends and thus the shapes of phase diagrams. Several facets of the general LCT become evident from the recently developed "lattice cluster theory for pedestrians" which provides extremely simple and physically instructive expressions for the effective Flory-Huggins parameter in the incompressible, high molecular weight limit. ${ }^{11}$ The relation of blend thermodynamic properties to monomer molecular structures is represented in these simple expressions by simple geometrical 
indices that count the number of ways of selecting bonded sequential units in structured monomer chains of the individual components. ${ }^{11}$ The "pedestrian" LCT has been extended to describe random copolymer systems with explicit applications to blends of saturated polybutadienes with different saturated vinyl contents. ${ }^{12}$ While this incompressible limit provides enormous physical insight and is useful for the analysis of certain experimental data, a theoretical description of the pressure dependence requires the use of the full compressible version of the LCT. Moreover, the experiments are confined to rather low molecular weight $\mathrm{dPB} / \mathrm{PS}$ samples because of the rather strong immiscibility of PB and PS. Thus, the full LCT is necessary for the analysis of the experiments for these low molecular weight $\mathrm{dPB} / \mathrm{PS}$ blends. While the full LCT has been derived for blends of homopolymers, the theory has only been developed for limited classes of random copolymer systems, e.g., when all monomers have vinyl structures and the polymers are taken as completely flexible. These restrictions preclude describing the PB samples which are all random copolymers due to varying degrees of 1,2 vs 1,4 addition units and due to the presence of cis and trans 1,4 units. Thus, the various PB samples are modeled here as pure homopolymers to elucidate some general trends in the pressure dependence rather than to achieve a quantitative fit to the experimental data. Another strong impediment to a quantative representation of the experiments is associated with the low molecular weights of the blend components that contain chemically distinct end groups whose thermodynamic effects have recently been shown by two of us as being non-negligible. ${ }^{13}$

The lattice cluster theory also describes the influence of chain semiflexibility on the properties of polymer blends. ${ }^{14}$ The variation of blend thermodynamic properties and miscibility with chain semiflexibility is explained in the LCT by using a few rather simple effective geometrical parameters that depend on the monomer molecular structures and on the energy differences between trans and gauche conformations. The present comparisons of LCT calculations with SANS data demonstrate that the inclusion of chain semiflexibilty into the LCT significantly improves the ability of the theory to describe the temperature, pressure, and microstructure dependence of the SANS effective FH parameter.

Section II outlines both the crossover theory used to extract mean-field-limiting information from the experimental SANS data and some basic facets of the LCT. The experimental Sec. III is followed by a description of the results in Sec. IV. The experiments are compared with the LCT for the temperature, pressure, microstructure, and chain stiffness dependence of the effective Flory interaction parameter.

\section{THEORY}

This section briefly summarizes the theory used for describing the thermodynamics of polymer blends and for comparing with the experimental data that cover regions in which there are strong composition fluctuations (near the critical point) and weak composition fluctutions (far from the critical point), as well as the crossover region in between. Since contributions from long-range fluctuations are absent from the mean-field LCT that is employed to analyze the blend pressure dependence, the comparison of experiment and theory requires extracting the mean-field behavior of the zero-angle scattering, the susceptibility $S(0)$ from the experimental data for $S(0)$ by using an additional theory that describes the general variation of the susceptibility $S(0)$ in all regions, including the region near the critical point, where three-dimensional (3D) Ising behavior is observed, and the mean-field region far from the critical point.

The susceptibility is represented in terms of an effective interaction parameter, namely, the SANS Flory-Huggins parameter $\Gamma$. The present experiments for PB/PS blends indicate that $\Gamma$ for these systems has the customary representation $\Gamma=-\Gamma_{\sigma}+\Gamma_{h} / T$ in terms of entropic $\Gamma_{\sigma}$ and enthalpic $\Gamma_{h}$ contributions. The lattice cluster theory provides an analytical expression for the free energy of the binary blend, and, as shown in Sec. II D 4, the interaction parameter $\Gamma$ may readily be evaluated from this free energy. The LCT is based on the extended lattice model in which individual monomers of both blend components are endowed with molecular structures and, therefore, may occupy several lattice sites. The description of the pressure dependence of $\Gamma$ requires the inclusion of excess free volume into the extended lattice model, and the fraction of vacant sites is determined from the equation of state. (The "voids" are not, however, separate thermodynamic species.) Particularly, simple and illuminating expressions emerge from the LCT in the high pressure, high molecular weight, fully flexible chain limit, so this limit is briefly discussed to explain certain facets of the theory, such as the molecular origins of the entropic and enthalpic portions of $\Gamma .{ }^{11}$

\section{A. Susceptibility}

The critical behavior of thermal composition fluctuations in polymer blends above the critical point can be probed by neutron scattering experiments. The scattering contrast between the two blend components is rendered large by the deuteration of one component. Measurements of the composition fluctuations yield the structure factor $S(Q)$ as a function of the scattering vector $Q$. Within the Ornstein-Zernicke approximation, the structure factor is given by

$$
S(Q)=\frac{S(0)}{1+\xi^{2} Q^{2}},
$$

with the correllation length $\xi$. The form of Eq. (1) is used to extrapolate experimental data to $Q=0$. According to the fluctuation-dissipation theorem, ${ }^{1}$ the susceptibility $S(0)$ is related to the second derivative of the Gibbs free energy with respect to the blend composition $\Phi$ (say, the volume fraction of species 1),

$$
S^{-1}(0)=\left.\frac{1}{V R T} \frac{\partial^{2} G}{\partial \Phi^{2}}\right|_{P, T},
$$

where $V$ is the volume of the sample and $R T$ is the molar thermal energy. The susceptibility $S(0)$ behaves differently in the critical and mean-field regimes as described by two separate universality classes. ${ }^{3}$ In the vicinity of the critical point, the susceptibility displays three-dimensional Ising behavior, which implies 


$$
S(0)^{-1}=C_{+}^{-1} \tau^{\gamma},
$$

with $C_{+}$the critical amplitude, the reduced temperature $\tau$ defined as $\tau=\left|1-T_{c} / T\right|$, and with the 3D Ising critical exponent $\gamma=1.239$. At temperatures far from the critical temperature $T_{c}$, mean-field behavior is observed with the characteristic mean-field critical exponent $\gamma$ of 1 and with the mean-field $(\mathrm{MF})$ critical amplitude $C_{\mathrm{MF}}$ replacing $C_{+}$in Eq. (3). This $C_{\mathrm{MF}}$ must be extracted from the experimental data for $S(0)$ in order to ensure a meaningful comparison with mean field theories for polymer blends.

\section{B. Crossover function}

A crossover function represents the susceptibility over the whole temperature range, thereby connecting the behaviors in the two distinct universality classes and enabling us to extract the mean-field susceptibility for comparison with the mean-field LCT. We describe the measured $S(0)$ by a crossover function derived by Belyakov and Kiselev, ${ }^{15}$ which can be written as

$$
\begin{aligned}
\hat{\tau}= & {\left[1+2.333 \hat{S}(0)^{\Delta / \gamma}\right]^{(\gamma-1) / \Delta} } \\
& \times\left\{\hat{S}(0)^{-1}+\left[1+2.333 \hat{S}(0)^{\Delta / \gamma}\right]^{-\gamma / \Delta}\right\} .
\end{aligned}
$$

The functional form in Eq. (4) represents the renormalized reduced temperature $\hat{\tau}=\tau / G i$ as a function of the renormalized susceptibility $\hat{S}(0)=S(0) \mathrm{Gi} / C_{\mathrm{MF}}$ where Gi denotes the Ginzburg number. The latter quantity is proportional to the reduced temperature at the boundary between the mean-field and the crossover ranges, ${ }^{4}$ and explicit definitions of $\mathrm{Gi}$ are presented in Ref. 16 along with calculations of Gi based on the LCT. The exponents $\gamma=1.239$ and $\Delta=0.51$ apply for 3D Ising critical behavior. Although the crossover function in Eq. (4) has been derived by renormalization group methods only to first order in the parameter $\epsilon=4-d$, with $d$ the dimensionality of the system, we follow the phenomenological approach that entails using the exact critical exponents of the 3D Ising model in place of the first-order approximations. ${ }^{17}$

\section{Mean-field behavior}

The description of the crossover between critical and mean-field behavior of polymer blends employs LandauGinzburg-Wilson theory ${ }^{17}$ in which the Gibbs free energy $G$ is expanded in terms of the order parameter $\Psi(r)=\phi(r)$ $-\phi_{C}$ where $\phi(r)$ is the actual volume fraction of species 1 at the spacial position $r$ and $\phi_{C}$ is its value at the critical point. The mean-field approximation is valid as long as the fluctuations $\delta \Psi(r)=\Psi(r)-\Psi$ of the order parameter are negligibly small (with $\Psi \equiv\langle\Psi\rangle$ being the average). When the composition fluctuations become relevant (i.e., near the critical point), $G$ is given by the form used in renormalization group theories,

$$
\begin{aligned}
\frac{G}{V R T}= & \int d^{3} r\left\{\frac{1}{2 !} a_{0} \tau^{\prime} \Psi^{2}(r)+\frac{1}{4 !} u_{0} \Psi^{4}(r)\right. \\
& \left.+\frac{1}{2 !} c_{0}[\nabla \Psi(r)]^{2}\right\},
\end{aligned}
$$

which can treat systems with strong critical fluctuations as well as the crossover to mean-field behavior. The coefficients $a_{0}, u_{0}$, and $c_{0}$ may be computed from a mean-field approximation as in Refs. 16 and 18. The critical mean-fieldamplitude $C_{\mathrm{MF}}$ is related to the coefficient $a_{0}$ by $a_{0}$ $=1 / C_{\mathrm{MF}}$ and within the extended lattice model is equal to

$$
C_{\mathrm{MF}}=\frac{1}{2} /\left(\Gamma_{\sigma}+\Gamma_{c}\right) \text {, }
$$

where $\Gamma_{\sigma}$ is the entropic portion of the effective interaction parameter as described below, and the critical value $\Gamma_{c}$ $\left.=(1 / 2) /\left\{\left(\phi V_{1}\right)^{-1}+\left[(1-\phi) V_{2}\right)\right]^{-1}\right\}$ of the interaction parameter $\Gamma$ is determined in terms of individual component molar volumes $V_{i}$. The mean-field-reduced temperature $\tau^{\prime}$ is defined as $\tau^{\prime}=\left|1-T_{c}^{\mathrm{MF}} / T\right|$, and the mean-field temperature $T_{c}^{\mathrm{MF}}$ is related to the actual critical temperature $T_{c}$ by

$$
T_{c}^{\mathrm{MF}}=T_{c} /(1-G i)=\Gamma_{h} /\left(\Gamma_{\sigma}+\Gamma_{c}\right) .
$$

Equation (7) reflects the fact that the "actual" critical temperature $T_{c}$ is lowered by critical fluctuations from the meanfield estimation. This means that the strong critical fluctuations introduce an additional contribution to the entropy of the system. Once the critical amplitude $C_{\mathrm{MF}}$ is known from fits of the experimental susceptibility $S(0)$ to the crossover function of Eq. (4), Eq. (6) enables evaluating the entropic part $\Gamma_{\sigma}$ of $\Gamma$, while Eq. (7) yields $\Gamma_{h}$. Equation (7) also provides a means for determining the mean-field critical temperature $T_{c}^{\mathrm{MF}}$ from the experimental data.

\section{Lattice cluster theory}

The lattice cluster theory (LCT) (Ref. 6) is based on a far more accurate mean-field solution of the lattice model for polymer systems than Flory-Huggins theory, as well as on the use of an extended lattice model in which monomers are endowed with molecular structures.

\section{Generalized lattice model of binary polymer blends}

The generalized lattice model represents a binary polymer blend by a set of $n_{1}$ and $n_{2}$ polymer chains of species 1 and 2 , respectively, placed on a regular array with $N_{l}$ lattice sites and coordination number $z$. Each monomer of a given species $i$ has a specified molecular structure and occupies $s_{i}$ $(i=1,2)$ lattice sites. The structures for both types of monomers are chosen, whenever possible, as those most closely corresponding to united atom models in which the united atom groups, such as $\mathrm{CH}_{n}$ groups, reside at single lattice sites. Figure 1 displays the monomer architectures used in our calculations. All chains are assumed to be monodisperse, for simplicity. Each homopolymer chain of species $i$ has $N_{i}$ monomers and extends over $M_{i}=N_{i} s_{i}$ lattice sites. The compressibility of the polymer blend is represented in the generalized lattice model by allowing $n_{v}$ lattice sites to be empty. The resulting volume fraction of excess free volume $\phi_{v}$ $=n_{v} / N_{l}$ is determined from the equation of state for a given pressure, temperature, blend composition $\Phi_{1}=1-\Phi_{2}$, and choice of the volume $v_{\text {cell }}^{\text {(blend }}$ of a unit cell on the lattice. The composition of the compressible binary polymer mixture may be described alternatively in terms of the actual volume fractions $\phi_{i}=n_{i} M_{i} / N_{l}$ [normalized as $\phi_{1}+\phi_{2}=1-\phi_{v}$ and related to the nominal volume fractions $\Phi_{i}$ as $\phi_{i}=\Phi_{i}(1$ 


\section{:3:}

PS

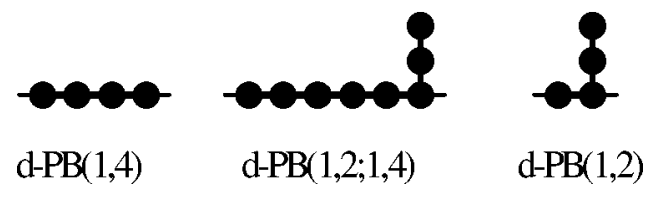

FIG. 1. United atom group models for polystyrene, 1,4 polybutadiene, 1,2 polybutadiene, and 1,4;1,2 polybutadiene monomers. The random copolymer 1,4;1,2 PB is modeled as a homopolymer with the averaged monomer structure having both 1,4 and 1,2 units as indicated in the figure.

$\left.-\phi_{v}\right)$ ]. The lattice is assumed to be a three-dimensional ( $d$ $=3)$ cubic lattice with $z=2 d=6$. The unit cell volume $v_{\text {cell }}^{\text {(blend) }}$ associated with each lattice site is evaluated by using a common combining rule (see Sec. V) which expresses $v_{\text {cell }}^{\text {(blend) }}$ as a function of the pure component volumes $v_{\text {cell }}^{(1)}$ and $v_{\text {cell }}^{(2)}$ and the blend composition $\Phi_{1}$. The pure melt volumes $v_{\text {cell }}^{(i)}$ are determined from fits to $P V T$ data. Interactions generally involve short-range repulsions and longer-range attractions. While the former are naturally represented in the lattice model by the excluded volume constraints that prohibit multiple occupancy of lattice sites, the latter are introduced by ascribing an attractive microscopic van der Waals energy $\epsilon_{\alpha \beta}^{(k, l)}$ to nearest-neighbor (on the lattice) portions $k$ and $l$ of monomers $\alpha$ and $\beta$. For simplicity, all $s_{\alpha}$ portions of a monomer of species $\alpha$ are taken as energetically equivalent units, which interact with the same energies $\epsilon_{\alpha \beta}$. This simplification leads for compressible binary blends to the presence of three independent interaction energies $\epsilon_{A A}, \epsilon_{B B}$, and $\epsilon_{A B}$ which are determined from experimental data. Pure melt $P V T$ data provide the input for specifying the single component $\epsilon_{A A}$ and $\epsilon_{B B}$, whereas $\epsilon_{A B}$ is obtained from fitting binary blend experimental data with the LCT.

\section{Free energy of binary polymer blends}

The LCT derives the Helmholtz free energy $F_{\text {blend }}$ for a binary blend as a perturbative expansion about a compressible generalization of the FH free energy $F_{\text {blend }}^{\mathrm{FH}}$. The LCT free energy $F_{\text {blend }}$ can be written formally as

$$
\frac{F_{\text {blend }}}{N_{l} k T}=\frac{F_{\text {blend }}^{\mathrm{FH}}}{N_{l} k T}+\text { corrections, }
$$

where the corrections to the compressible system generalization of the $\mathrm{FH}$ approximation $F_{\text {blend }}^{\mathrm{FH}}$ in Eq. (8) are derived as polynomials in the volume fractions $\phi_{1}$ and $\phi_{2}$, and $N_{l}$ is the total number of lattice sites. The coefficients of these polynomials are generated as double expansions in powers of the inverse lattice coordination number $1 / z$ and in powers of the dimensionless microscopic van der Waals energies $\left\{\epsilon_{\alpha \beta} / k T\right\}$. The coefficients in these double expansions depend, in turn, on the monomer structures of the blend components. An additional dependence of $F_{\text {blend }}$ on the trans- gauche conformational energy differences $\epsilon_{\text {bend }}^{(1)}$ and $\epsilon_{\text {bend }}^{(2)}$ emerges when chains of species 1 and 2 are treated as semiflexible. (The fully flexible chain system corresponds to $\epsilon_{\text {bend }}^{(1)}=\epsilon_{\text {bend }}^{(2)}=0$.) The introduction of polymer chain semiflexibility for a binary polymer blend, thus, implies the presence of two extra adjustable parameters $\epsilon_{\text {bend }}^{(1)}$ and $\epsilon_{\text {bend }}^{(2)}$. The technical details and the diagrammatic representation of $F_{\text {blend }}$ for both fully flexible and semiflexible chain blends are described in a series of papers. ${ }^{14,19-21}$ A similar expression for the free energy of a melt follows directly from Eq. (8) by setting $\phi_{1}=\phi$ and $\phi_{2}=0$. When, the expression in Eq. (8) is specialized to a pure homopolymer melt, only a single homocontact interaction energy $\epsilon_{\alpha \alpha}$ appears. The pressure $P$ is computed from Eq. (8) as

$$
P\left(T, \phi_{v}, v_{\text {cell }}^{\text {(blend) }}, \Phi_{1}\right)=-\left.\frac{\partial F_{\text {blend }}}{\partial V_{\text {blend }}}\right|_{T, n_{1}, n_{2}},
$$

or specializing to a melt (composed of $n$ polymer chains) as

$$
P\left(T, \phi_{v}, v_{\text {cell }}^{(\text {melt })}\right)=-\left.\frac{\partial F_{\text {melt }}}{\partial V_{\text {melt }}}\right|_{T, n} .
$$

The equations of state derived from Eqs. (9) and (10) are used to determine $\phi_{v}$ for binary blends and melts, respectively. Both derivatives of the free energy with respect to the volume are evaluated by taking $v_{\text {cell }}^{\text {(blen) }}$ and $v_{\text {cell }}^{\text {(melt) }}$ as independent of temperature and total volume.

\section{SANS interaction parameter $\Gamma$ for binary polymer blends}

Small-angle neutron scattering experiments for polymer systems directly provide the absolute scattering intensity $I(Q)=d \Sigma / d \Omega(Q)$. The standard analysis of the SANS data involves the extrapolation of $I(Q)$ to zero angle by the Ornstein-Zernicke approximation [Eq. (1)]. This analysis produces the effective Flory-Huggins interaction parameter $\Gamma$, an important quantity for characterizing and predicting thermodynamic properties of polymer systems. The present subsection describes how the scattering intensity $I(0)$ and the effective FH interaction parameter may be computed from the lattice cluster theory and the generalized lattice model. As shown in Refs. 10 and 22, the scattering intensity $I(0)$ can be expressed in terms of the partial structure factors $S_{i j}(0)$ (defined below on a per-monomer basis) and the monomer scattering length densities $b_{i}$ and $b_{j}$ by

$$
\begin{aligned}
I(0)= & \frac{\left(1-\phi_{v}\right)}{v_{\text {cell }}^{\text {(blend })}}\left[\frac{\Phi_{1}}{s_{1}}+\frac{\Phi_{2}}{s_{2}}\right] \\
& \times\left[b_{1}^{2} S_{11}(0)+b_{2}^{2} S_{22}(0)+2 b_{1} b_{2} S_{12}(0)\right],
\end{aligned}
$$

where $s_{i}$ designates the number of lattice sites occupied by a single monomer of species $i$ and where $\phi_{v}$ denotes the freevolume fraction. The zero-wave-vector limit of the partial structure factors $S_{i j}$ of Eq. (11) are obtained from the chemical potentials $\mu_{1}$ and $\mu_{2}$ by ${ }^{10}$

$$
S_{i j}(0)=\left.\frac{N_{i} N_{j} k_{B} T}{n_{1} N_{1}+n_{2} N_{2}} \frac{\partial n_{j}}{\partial \mu_{i}}\right|_{T, V, \mu_{k \neq i}} .
$$


The chemical potentials $\mu_{1}$ and $\mu_{2}$, in turn, are calculated from the free energy in Eq. (8) as

$$
\mu_{\alpha}=\left.\frac{\partial F}{\partial n_{\alpha}}\right|_{T, V, n_{\beta \neq \alpha}},
$$

where the volume $V$ is determined from the equation of state for given $P, T$, and $\Phi \equiv \Phi_{1}$. Hence, the use of the LCT free energy $F_{\text {blend }}$ and Eqs. (9)-(13) enables us to determine the absolute scattering intensity $I(0)$ from the lattice cluster theory. The LCT effective interaction parameter $\Gamma^{\mathrm{LCT}}$ is defined by the same formula that is used ${ }^{10}$ in the standard experimental RPA analysis of $I(0)$,

$$
\frac{k_{N}}{I(0)} \equiv \frac{1}{S(0)}=\frac{1}{N_{1} v_{1} \Phi_{1}}+\frac{1}{N_{2} v_{2} \Phi_{2}}-2 \Gamma^{\mathrm{LCT}},
$$

where

$$
k_{N}=N_{\mathrm{Av}}\left[b_{1} / v_{1}-b_{2} / v_{2}\right]^{2},
$$

is the scattering contrast factor, $N_{\mathrm{Av}}$ is Avogadro's number, and $v_{1}=V_{1} / N_{1}$ and $v_{2}=V_{2} / N_{2}$ are the molar monomer volumes for the pure components 1 and 2 .

The LCT interaction parameter $\Gamma^{\mathrm{LCT}}$ is compared to the experimental mean-field-limit SANS interaction parameter $\Gamma$. The latter quantity is extracted from the measured susceptibility $S(0)$ in the crossover region by fitting the crossover function of Eq. (4) to these measurements and by using Eqs. (6) and (7).

\section{LCT for pedestrians}

The analytical expressions for the LCT free energy and, therefore, for the effective interaction parameter $\Gamma^{\mathrm{LCT}}$ determined from Eq. (14) are very lengthy and are suitable only for numerical analysis. This complexity is an inevitable consequence of any theory that attempts to describe the combined influences of monomer structure, compressibility, and nonrandom mixing effects on the thermodynamic properties of polymer blends. We have recently shown, ${ }^{11,12}$ however, that the imposition of the high pressure, high molecular weight, fully flexible chain limit to the lattice cluster theory produces remarkably simple and compact equations that are useful for probing general trends and sometimes even for analyzing experimental data. The effective SANS interaction parameter $\Gamma^{\mathrm{LCT}}$ emerges for binary homopolymer blends from this pedestrian version of the LCT as the rather simple expression,

$$
\begin{aligned}
\Gamma^{\mathrm{LCT}}= & \frac{1}{v_{\text {cell }}^{\text {(blend })} N_{\mathrm{Av}}}\left\{\frac{\left(r_{1}-r_{2}\right)^{2}}{z^{2}}+\frac{\epsilon}{R T}\right. \\
& \left.\times\left(\frac{z-2}{2}+\frac{1}{z}\left\{p_{1}[1-3(1-\phi)]+p_{2}(1-3 \phi)\right\}\right)\right\}
\end{aligned}
$$

where $\epsilon=\epsilon_{A A}+\epsilon_{B B}-2 \epsilon_{A B}$ is the microscopic exchange energy and $r_{i}$ and $p_{i}(i=1,2)$ are geometrical indices that can be easily obtained from the monomer united atom structures. In particular, the structural parameter $r_{i}$ may be represented ${ }^{11}$ in terms of the respective numbers $s_{i}^{\text {(tri) }}$ and $s_{i}^{\text {(tetra) }}$ of tri- and tetrafunctional united atom groups in a single monomer of species $i$ as

$$
r_{i}=1+\frac{s_{i}^{\text {(tri) }}}{s_{i}}+3 \frac{s_{i}^{\text {(tetra) }}}{s_{i}},
$$

where $s_{i}$ designates the number of united atom units in an $i$ species monomer (coinciding with the number of lattice sites occupied by this monomer). The second geometrical index $p_{i}=N_{i}^{(3)} / M_{i}$ denotes the ratio of the number $N_{i}^{(3)}$ of distinct sets of three sequential bonds in a chain of species $i$ and the site occupancy index $M_{i}=N_{i} s_{i}$. The ratios $r_{i}$ and $p_{i}$ are listed in Ref. 11 for a wide range of monomer structures. The $\epsilon^{2}$ contribution in Eq. (16) is numerically negligible for binary homopolymer blends and, therefore, is omitted.

The first term in the braces on the right-hand side of Eq. (16) provides the athermal limit entropic component of $\Gamma^{\mathrm{LCT}}$ which depends on the monomer structures of the two blend components. This entropic portion of $\Gamma$ represents a correction to the $\mathrm{FH}$ combinatorial entropy that arises from the packing of chains with monomers of different sizes and shapes. The remaining contribution to $\Gamma^{\mathrm{LCT}}$ in Eq. (16) is of energetic origin and contains the composition-dependent contact probability for the two interacting monomer species. This term extends the "surface fraction" concept of Guggenheim to polymer chains with structured monomers, providing an explicit formula for computing this previously vague quantity.

\section{EXPERIMENTAL SECTION}

Protonated polystyrene and deuterated 1,4-polybutadiene $[d$-PB $(1,4)]$ have been synthesized from the respective styrene and butadiene monomers by anionic polymerization in benzene with the presence of sec-butyllithium as initiator. Syntheses of deuterated 1,2;1,4-polybutadiene $[d$-PB $(1,2 ; 1,4)]$ and deuterated 1,2-polybutadiene $[d$-PB $(1,2)]$ have been performed in diethyl ether and tetrahydrofurane, respectively, in order to control the vinyl content of PB. The 1,4 content of the polybutadiene samples is determined by ${ }^{13} \mathrm{C}$-NMR and is given in Table I along with the samples' molecular weights and polydispersities. The molecular weights and their distributions have been measured by vapor pressure osmometry and size exclusion chromatography, respectively. The binary mixtures are preparated by disolving polybutadiene and polystyrene (with $0.1 \%$ antioxidant) in benzene, and then by freeze drying the mixture. Table I summarizes the parameters characterizing the pure PB and PS melts, and the three blends having PS as a common component. The critical blend compositions $\Phi_{C}$ for the three binary systems have been determined from neutron scattering experiments and are listed in Table I (see also Ref. 3).

The neutron experiments have been performed at the small-angle neutron scattering facility KWS1 of the research reactor FRJ2 at the Forschungszentrum Jülich. A specially designed pressure/temperature cell enables in situ experiments over the pressure range of $0.1-200 \mathrm{MPa}$ and over the temperature range of $-20-200^{\circ} \mathrm{C}$, stabilized to within 
TABLE I. Characterization of the experimental samples of three PB/PS blends with varying microstructure of the $\mathrm{PB}$ component. The table also summarizes the blend critical compositions (as determined from SANS experiments), the microscopic van der Waals interaction energies $\epsilon_{i j}(i, j$ $=1,2)$, the exchange energies $\epsilon=\epsilon_{11}+\epsilon_{22}-2 \epsilon_{12}$, the bending energies $\epsilon_{\text {bend }}^{(i)}$, the unit cell volumes for the pure blend components $v_{\text {cell }}^{i}$, as well as the geometrical parameters $r_{i}$ and $p_{i}(i=1,2)$.

\begin{tabular}{lllll}
\hline & $d$-PB $(1,4)$ & $d$-PB $(1,2 ; 1,4)$ & $d$-PB $(1,2)$ & \multicolumn{1}{c}{ PS } \\
\hline$M_{N}(\mathrm{~g} / \mathrm{mol})$ & 2100 & 1900 & 2000 & $1700 ; 1800$ \\
$M_{W} / M_{N}$ & $<1.1$ & $<1.1$ & $<1.1$ & 1.06 \\
$N_{i}$ & 35 & $16(32)$ & 33 & $16 ; 17$ \\
1,4 content & $93 \%$ & $46 \%$ & $9 \%$ & $\ldots$ \\
$r_{i}$ & 1 & & & \\
$p_{i}$ & 1 & $9 / 8$ & $5 / 4$ & $9 / 7$ \\
$M_{i}$ & 140 & $10 / 8$ & $6 / 4$ & $14 / 7$ \\
$\epsilon_{i i} / k(\mathrm{~K})$ & $209.5 \pm 0.5$ & $210.5 \pm 0.5$ & $211.6 \pm 0.5$ & $234.0 \pm 0.5$ \\
$v_{\text {cell }}\left(\AA^{3}\right)$ & $23.40 \pm 0.01$ & $23.40 \pm 0.01$ & $23.37 \pm 0.01$ & $19.71 \pm 0.01$ \\
$\Phi_{C}(\mathrm{SANS})$ & 0.420 & 0.505 & 132 & $\ldots$ \\
$\epsilon_{\mathrm{PB}-\mathrm{PS}} / k(\mathrm{~K})$ & 220.13 & 220.95 & 220.16 & $\ldots$ \\
$\epsilon_{\mathrm{ex}} / k(\mathrm{~K})$ & $3.24 \pm 0.26$ & $2.60 \pm 0.44$ & $5.28 \pm 0.20$ & $\ldots$ \\
$\epsilon_{\text {bend }} / k(\mathrm{~K})$ & $1000 \pm 50$ & $1250 \pm 50$ & $1500 \pm 50$ & 3000 (set) \\
\hline \hline
\end{tabular}

$\leqslant 0.01 \mathrm{~K}$. The sample thickness is $1 \mathrm{~mm}$, and its aperture has a diameter of $7 \mathrm{~mm}$. The neutron wave length equals $7 \AA$ with a distribution of $\pm 10 \%$. The scattered intensity is corrected to remove the background contribution and is converted to absolute units using a lupolene standard. Within the traditional incompressible RPA model for analysis, the structure factor $S(Q)$ is related to the macroscopic cross section by $d \Sigma / d \Omega(Q)=k_{N} S(Q)$, where the contrast factor $k_{N}$ involves the difference in the scattering length densities of the two blend components, as given by Eq. (15). Other details are described in Ref. 3.

The equation of state properties of the pure blend components have been measured at the Gnomix facility of the MPI at Mainz. The experiments provide the specific volumes (relative to a reference value at $t=20^{\circ} \mathrm{C}$ and $P=0.1 \mathrm{MPa}$ ) as a function of temperature and pressure over the range of 10-200 MPa in $5 \mathrm{MPa}$ increments. The piezometer cell contains a sample of about $1 \mathrm{~g}$, and is filled with mercury. Volume deviations are measured by the excursions of the metal bellows of the cell.

\section{RESULTS}

The LCT computations of $\Gamma^{\mathrm{LCT}}$ have been performed for three $\mathrm{PB} / \mathrm{PS}$ blends $[\mathrm{PB}(1,4) / \mathrm{PS}, \quad \mathrm{PB}(1,2) / \mathrm{PS}$, and $\mathrm{PB}(1,2 ; 1,4) / \mathrm{PS}]$ varying with the vinyl content of the $\mathrm{PB}$ component. Figure 1 depicts the monomer structures for the PB and PS polymer species. The polystyrene monomer is mimicked with two united atom groups lying on the chain backbone and the H-shaped side group composed of six united atom groups, as in phenyl groups, leading to the total number of occupied lattice sites as $s=8$. This simplifying model of the styrene ring is introduced because the currently available LCT does not apply to systems of flexible chains with closed-loop side groups. The 1,4-polybutadiene $[\mathrm{PB}(1,4)]$ is represented by a linear homopolymer containing four united atom groups per monomer $(s=4)$, while the 1,2- polybutadiene $[\mathrm{PB}(1,2)]$ is modeled by a branched homopolymer chain having one vinyl group per monomer and the same monomer occupancy index $s=4$. The 1,2;1-4 polybutadiene is truly a random copolymer of 1,2- and 1,4butadiene units. For simplicity, however, we treat this random copolymer as a homopolymer with an averaged monomer structure comprising both 1,4 and 1,2 units and extending over $s=8$ lattice sites. The resulting geometrical parameters $r_{i}$ and $p_{i}$ for three different model PB chains and PS are presented in Table I. The chain occupancy index $M_{i}$ for a given polymer species is determined as the product of its polymerization index $N_{i}$ and monomer site occupancy index $s_{i}$. Table I indicates that the nominally 1,2 and 1,4 PB samples contain a small degree of the 1,4 and 1,2 microstructures, respectively, implying that these systems, in reality, are likewise random copolymers and limiting the accuracy by which LCT computations for a homopolymer blend can model these systems. The presence of both cis and trans 1,4 addition isomers adds to this randomness and represents an additional impediment to a quantitative representation of the data with the LCT free-energy expressions appropriate to a model of these systems as pure homopolymers. In addition, the sec buthyl end groups are chemically distinct (different interaction energies and molecular structures) from each interior chain unit. Because the poor miscibility of PB and PS requires the use of low molecular weight samples, these end groups provide thermodynamically relevant contributions which are totally ignored by the LCT theory within a pure homopolymer blend model. Thus, our goal in comparing experiment and theory is only to reproduce the general pressure dependence and thereby provide insights into molecular factors affecting the variations of $\Gamma_{\sigma}$ and $\Gamma_{h}$ with pressure (as well as with microstructure). As already mentioned, the LCT for binary blends of fully flexible chains contains five adjustable energy parameters: three microscopic interaction energies $\epsilon_{11}, \epsilon_{22}$, and $\epsilon_{12}$ and two cell volumes $v_{\text {cell }}^{(1)}$ and $v_{\text {cell }}^{(2)}$ (associated with a single united atom group in each pure blend component). ${ }^{10}$ The self-interaction energies $\epsilon_{11}$ and $\epsilon_{22}$ and the cell volumes $v_{\text {cell }}^{(1)}$ and $v_{\text {cell }}^{(2)}$ are fit to the pure component $P V T$ data.

The blend unit cell volume $v_{\text {cell }}^{\text {(blend }}$ is evaluated by applying a common combining rule ${ }^{10}$

$$
\begin{aligned}
v_{\text {cell }}^{(\text {blend })}= & v_{\text {cell }}^{(1)} \Phi_{1}^{2}+v_{\text {cell }}^{(2)} \Phi_{2}^{2}+(1 / 4) \\
& \times\left[\left(v_{\text {cell }}^{(1)}\right)^{1 / 3}+\left(v_{\text {cell }}^{(2)}\right)^{1 / 3}\right]^{3} \Phi_{1} \Phi_{2},
\end{aligned}
$$

while the microscipic heterocontact interaction energy $\epsilon_{12}$ is determined from fits to binary blend data as described below. We begin describing our results with a brief analysis of these fits to pure component data.

Figure 2 displays the fit of the LCT equation of state to the experimental $p V T$ data for the polystyrene melt. The fit involves only two adjustable parameters $\left(\epsilon_{\mathrm{PS}-\mathrm{PS}}\right.$ and $\left.v_{\text {cell }}^{(\mathrm{PS})}\right)$ as opposed to equation of state theories that generally contain three parameters, at minimum. The glass transition limits the temperature range over which the fits may be made, with the glass-transition temperature increasing as usual with pressure. Theory and experiment agree for intermediate pressure ranges, while deviations appear at low and high pres- 


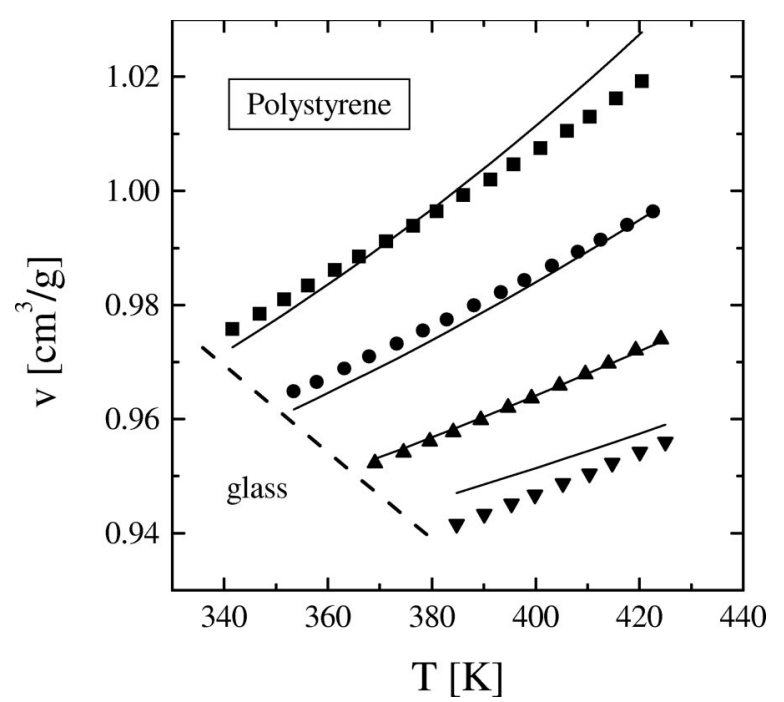

FIG. 2. Specific volume of polystyrene as a function of temperature for different pressures of $10 \mathrm{MPa}(\boldsymbol{\square}), 50 \mathrm{MPa}(\boldsymbol{\Delta}), 100 \mathrm{MPa}(\boldsymbol{\Delta})$, and 150 $\operatorname{MPa}(\boldsymbol{\nabla})$. Solid lines indicate the lattice cluster theory fits. Dashed line demarks the glass transition.

sures. As is common with equation of state theories, very small adjustments in the interaction energy and cell volume can produce improved fits of theory to experiment in the lower or higher pressure ranges. In addition, it is perfectly possible and perfectly reasonable to introduce an additional parameter to represent the thermal expansion of $v_{\text {cell }}^{(\mathrm{PS})}$ and thereby to generate improved fits, but this is not done here to avoid the use of additional parameters. The experimental $p V T$ data for the three polybutadienes with varying vinyl content appear similar to each other (see Fig. 3, where solid curves describe the LCT fits). The pure component interaction energies $\epsilon_{i i}$ and the cell volumes $v_{\text {cell }}^{(i)}$ for the different $d$-PB samples are almost identical to each other, as shown in Table I. As discussed below, the parameters $\epsilon_{i i}$ and $v_{\text {cell }}^{(i)}$ in the LCT equation of state are not significantly affected by the choice of the bending energy over rather wide ranges of possible blending energies.

Table I presents all the adjustable parameters. In fitting the $p V T$ data, we have also investigated the sensitivity of $\epsilon_{\mathrm{PS}-\mathrm{PS}}$ and $v_{\text {cell }}^{(\mathrm{PS})}$ to the assumed degree of chain stiffness. Only a very minor dependence is found when using rather extreme values for bending energies $\left(\epsilon_{\text {bend }} / k \leqslant 3000 \mathrm{~K}\right)$. This very weak dependence can be explained by the fact that the leading contribution to the LCT free energy is proportional to the product $\phi_{1}^{2} \epsilon_{\mathrm{PS}-\mathrm{PS}}$ (where $\phi_{1}^{2}$ is the lowest-order approximation to the probability of united atom group contacts), which to lowest order is not explicitly dependent on the bending energy. In the following, we neglect any dependence of $\epsilon_{i i}$ and $v_{\text {cell }}^{(i)}$ on the bending energy.

Having fit the homocontact interaction energies $\epsilon_{\mathrm{PS}-\mathrm{PS}}$ and the $\epsilon_{\mathrm{PB}-\mathrm{PB}}$, the heterocontact energy $\epsilon_{\mathrm{PS}-\mathrm{PB}}$ is determined by comparing LCT computations of $\Gamma^{\mathrm{LCT}}$ with the mean-field experimental FH parameter, which is extracted from the SANS experiments. Figure 4 illustrates the experimental reciprocal susceptibility $S(0)^{-1}$ for the $d$-PB $(1,4) / \mathrm{PS}$ blend as a function of temperature and pressure. The recip-
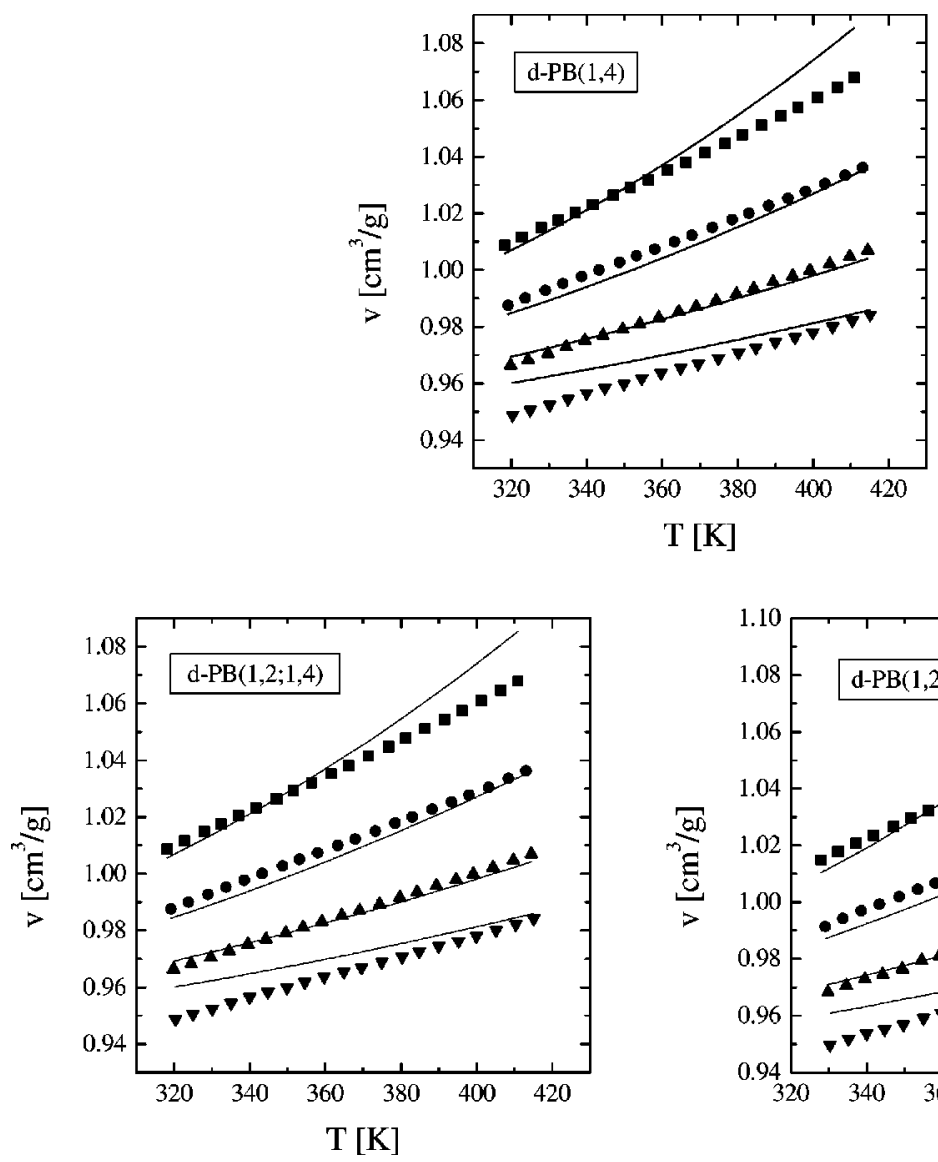

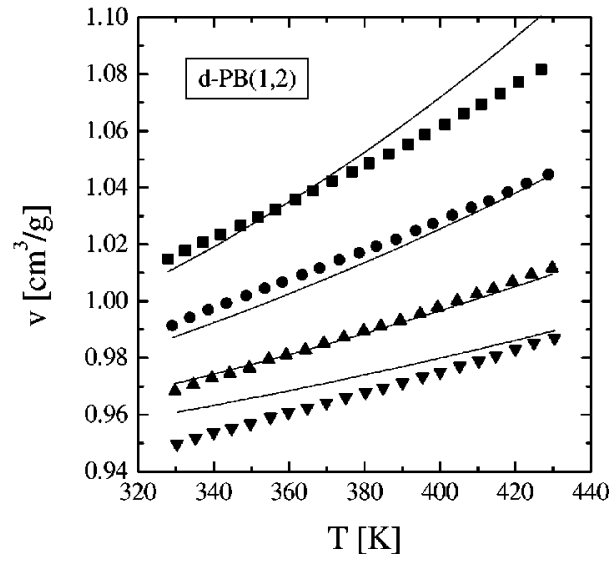

FIG. 3. Specific volume of deuterated polybutadienes with different microstructures as a function of temperature for pressures of $10 \mathrm{MPa}(\boldsymbol{\square}), 50 \mathrm{MPa}$

(•), $100 \mathrm{MPa}(\mathbf{\Delta})$, and $150 \mathrm{MPa}$ $(\boldsymbol{\nabla})$. Lines indicate the fits of the LCT equation of state to the experimental $P V T$ data. 


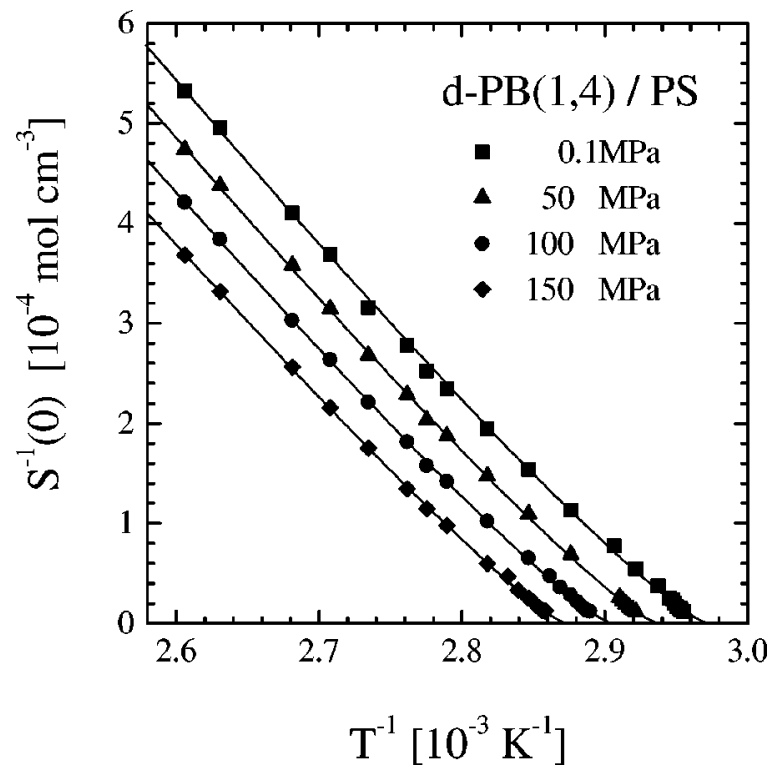

FIG. 4. Inverse susceptibility $S(0)^{-1}$ of the $d$-PB $(1,4) / \mathrm{PS}$ blend at the critical blend composition $\Phi_{1}=\Phi_{c}(\mathrm{~PB})=0.42$ as a function of temperature and pressure. Solid lines represent the fits to the crossover function of Eq. (4), and the symbols indicate the experimental data.

rocal susceptibility diminishes with decreasing temperature and with increasing pressure, thereby signaling the approach to the spinodal. The solid lines represent fits of the crossover function from Eq. (4) to the experimental data for $S(0)^{-1}$. The deviation from linearity of the $S(0)^{-1}(T)$ curves decreases when the pressure is increased, indicating that the Ginzburg number is diminished and that the critical region has reduced extent. Fitting the experimental data to the crossover function yields the Ginzburg number $\mathrm{Gi}$, the critical amplitude $C_{\mathrm{MF}}$, and the critical temperature $T_{C}$ as a function of pressure. The mean-field effective SANS interaction parameter $\Gamma$ is then obtained by transforming Eq. (7) to

$$
\tau=1-(1-\mathrm{Gi}) \frac{\Gamma+\Gamma_{\sigma}}{\Gamma_{C}+\Gamma_{\sigma}},
$$

and finally solving for $\Gamma$,

$$
\Gamma=\frac{\mathrm{Gi}}{1-\mathrm{Gi}} \frac{1-\hat{\tau}}{2 C_{\mathrm{MF}}}+\Gamma_{C},
$$

where the reduced temperature $\hat{\tau}=\tau / \mathrm{Gi}$ is given by $\mathrm{Eq}(4)$. This procedure produces the effective SANS interaction parameter $\Gamma$ (which is free of contributions from long-range fluctuations) and is applied to each measured value of the susceptibility, assuming, of course, that the parameters $\mathrm{Gi}$, $C_{\mathrm{MF}}$, and $T_{C}$ are first determined from fits to experimental data. Thus, the number of relevant data points for $\Gamma$ is actually reduced by 3 , but this reduction is of minor consequence since it is small compared to the typically 20 measured data points.

A first comparison between theory and experiment in Fig. 5 describes the theoretical FH parameter over a wide range of pressures as computed using a model in which all chains are completely flexible, i.e., by setting the bending energies to zero. At this initial stage, the lattice cluster theory

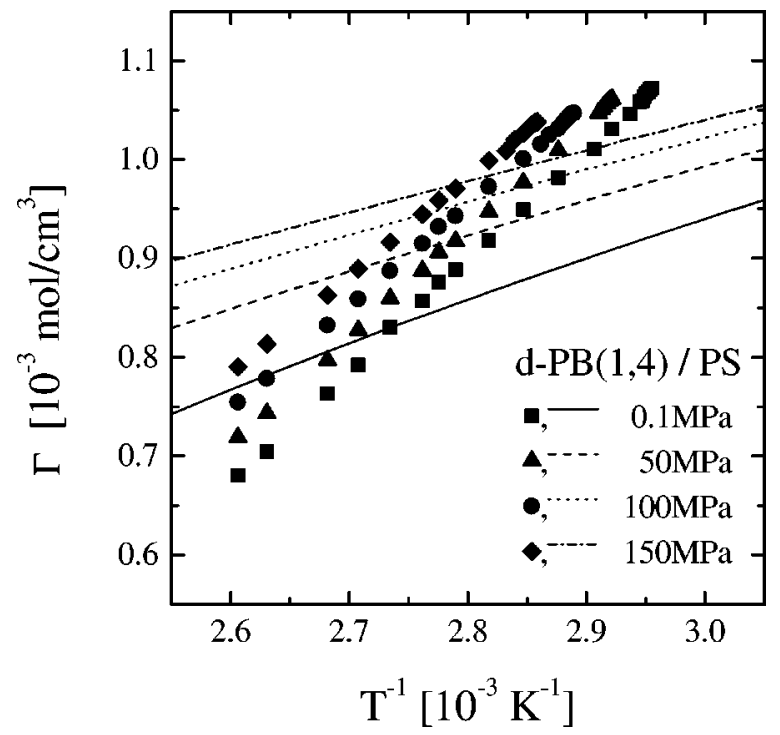

FIG. 5. LCT fits (lines) to the experimental effective interaction parameter $\Gamma$ (symbols) over wide ranges of temperatures and pressures for the $d$ - $\mathrm{PB}(1,4) / \mathrm{PS}$ blend at the critical composition $\Phi_{1}=\Phi_{c}(\mathrm{~PB})=0.42$. Both $\mathrm{PB}$ and PS chains are modeled in this figure as fully flexible $\left(\epsilon_{\text {bend }}^{(\mathrm{PB})}=\epsilon_{\text {bend }}^{(\mathrm{PS})}\right.$ $=0$ ). The only adjustable parameter of the theory is the heterocontact interaction energy $\epsilon_{12} / k=220.80 \mathrm{~K}$. The monomer scattering lengths $b_{\mathrm{PB}}$ and $b_{\mathrm{PS}}$ are taken as $b_{\mathrm{PB}}=6.66 \times 10^{-12} \mathrm{~cm}$ and $b_{\mathrm{PS}}=2.33 \times 10^{-12} \mathrm{~cm}$. The same values of $b_{\mathrm{PB}}$ and $b_{\mathrm{PS}}$ are employed in the fits presented in Figs. 6-8.

only roughly reproduces the effective SANS interaction parameter. Both enthalpic and entropic portions of $\Gamma$ depart strongly from the corresponding experimental values. The qualitative variations of both $\Gamma_{\sigma}$ and $\Gamma_{h}$ with pressure are determined, however, quite correctly, and this is the main goal of the theoretical analysis in view of the great simplifying assumptions outlined at the beginning of this section.

While introducing a bending energy does not appreciably alter the description of the pure melt $P V T$ data, the computed effective interaction parameter $\Gamma$ turns out to be very sensitive to the stiffness disparities between the two blend components. As illustated in Fig. 6, by including chain semiflexibility through introducing nonzero bending energies, the theoretical representation of the effective SANS interaction parameter is greatly improved. The improvement appears mostly in the enthalpic portion of $\Gamma$. The adjustable parameters are summarized in Table I. The examples in Fig. 6, as those in Fig. 5, refer to the $d$-PB(1,4)/PS mixture. The theoretical fits for the samples $\mathrm{PB}(1,2 / 1,4) / \mathrm{PS}$ and $\mathrm{PB}(1,2) / \mathrm{PS}$ are of a similar quality to those for the $\mathrm{PB}(1,4) / \mathrm{PS}$ blend and are shown in Figs. 7 and 8, respectively. Note again that the heterocontact $\epsilon_{\mathrm{PB}-\mathrm{PS}}$ interaction energies are almost independent of microstructure as found above for the pure component interactions $\epsilon_{\mathrm{PB}-\mathrm{PB}}$. The bending energies, a measure of the degree of steric interactions, increase, as expected, with the fraction of branched 1,2 PB units. The PS monomer is assumed to be even stiffer.

\section{DISCUSSION}

Small-angle neutron scattering measurements for three binary $\mathrm{dPB} / \mathrm{PS}$ blends of varying microstructure are combined with lattice cluster theory (LCT) calculations to probe 


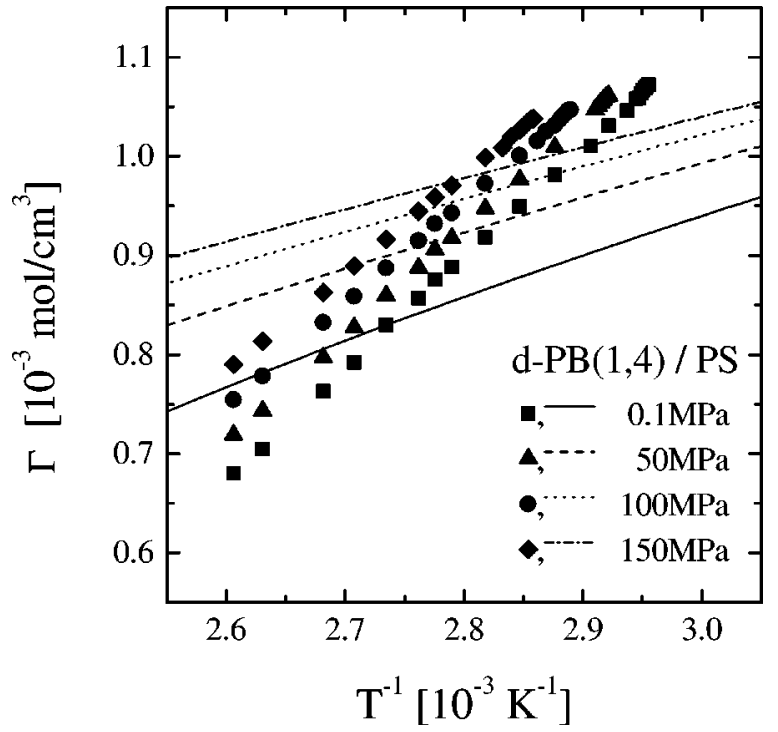

FIG. 6. LCT fits (lines) to the experimental effective interaction parameter $\Gamma$ (symbols) over wide ranges of temperatures and pressures for the $d$ - $\mathrm{PB}(1,4) / \mathrm{PS}$ blend at the critical composition $\Phi_{1}=\Phi_{c}(\mathrm{~PB})=0.42$. The bending energies are $\epsilon_{\text {bend }}^{(\mathrm{PS})} / k=3000 \mathrm{~K}$ and $\epsilon_{\text {bend }}^{(\mathrm{PB})} / k=1000 \mathrm{~K}$, and the heterocontact interaction energy is $\epsilon_{12} / k=220.13 \mathrm{~K}$, providing the three adjustable parameters of the theory as generated from least square fits.

the pressure, temperature, and microstructure dependence of the basic Flory-Huggins effective interaction parameter $\Gamma$. Since the SANS measurements yield extrapolated zero-angle susceptibilities $S(0)$ that contain contributions from longrange correlations, it is necessary to remove from the measured $S(0)$ the portions that stem from long-range correlations before analyzing the experimental data with mean field theory which, by definition, omits the non-negligible contributions from long-range correlations. Thus, the experimental

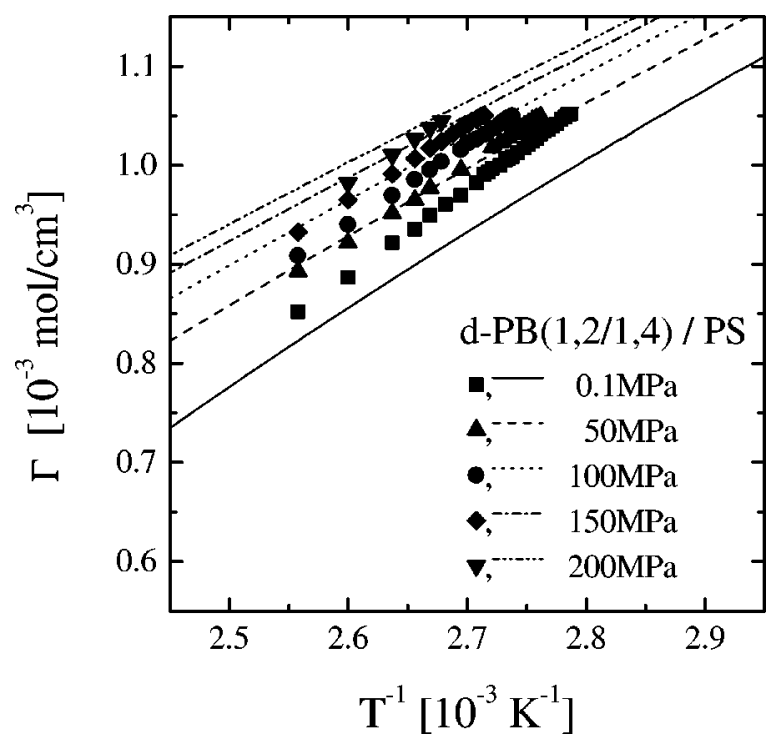

FIG. 7. LCT fits (lines) to the experimental effective interaction parameter $\Gamma$ (symbols) over wide ranges of temperatures and pressures for the $d$ - $\mathrm{PB}(1,2 ; 1,4) / \mathrm{PS}$ blend at the critical composition $\Phi_{1}=\Phi_{c}(\mathrm{~PB})=0.505$. The bending energies obtained from least-square fits are $\epsilon_{\text {bend }}^{(\mathrm{PB})} / k=1250 \mathrm{~K}$ and $\epsilon_{\mathrm{bend}}^{(\mathrm{PS})} / k=3000 \mathrm{~K}$. The third adjustable parameter, the heterocontact interaction energy is $\epsilon_{12} / k=220.95 \mathrm{~K}$.

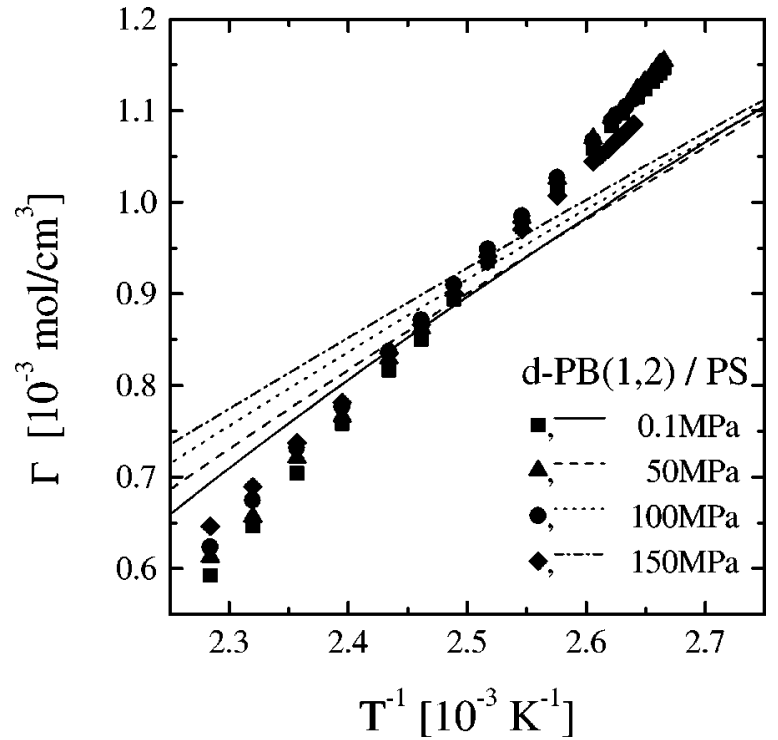

FIG. 8. LCT fits (lines) to the experimental effective interaction parameter for the $d-\mathrm{PB}(1,2) / \mathrm{PS}$ blend at the critical composition $\Phi_{1}=\Phi_{c}(\mathrm{~PB})=0.50$. The bending energies are $\epsilon_{\mathrm{bend}}^{(\mathrm{PB})} / k=1500 \mathrm{~K}$ and $\epsilon_{\mathrm{bend}}^{(\mathrm{PS})} / k=3000 \mathrm{~K}$, and the heterocontact interaction energy is $\epsilon_{12} / k=220.16 \mathrm{~K}$.

$S(0)$ are first fit to a crossover function to enable the direct determination of both the temperature-independent and temperature-dependent portions of the mean-field effective interaction parameter $\Gamma$. The LCT is chosen for the comparison with experiment because a thorough analysis of the pressure dependence imposes the need for using a theory that describes the systems as compressible and because a consideration of the variation of $\Gamma$ with $\mathrm{dPB}$ microstructure requires a theory that explicitly distinguishes between the differing molecular structures of the three PB samples. The lattice cluster theory satisfies these two essential requirements and has already been applied successfully to a wide variety of polymer blends. . $^{10,11,23,24}$

The LCT predicts the pressure dependence of $\Gamma^{\mathrm{LCT}}$ by including the presence of excess free volume into the underlying extended lattice model. The volume fraction of this excess free volume is determined from the equation of state for a given pressure, temperature, and blend composition. In accord with standard procedures in mixture theories, the comparison between theory and experiment begins with a fit of the homocontact interaction energies $\varepsilon_{i i}$ and the cell volumes $v_{\text {cell }}^{(i)}$ to pure component $p V T$ data. These fits for three binary mixtures of polystyrene and polybutadiene (with varying microstructure) produce almost identical values for the $\varepsilon_{\mathrm{PB}-\mathrm{PB}}$ and $v_{\text {cell }}^{(\mathrm{PB})}$ for these three systems, with the microstructure-induced differences in the experimental equations of state described within the theory through a series of monomer structure dependent structural indices (and differences in chain stiffness). The heterocontact interaction energy $\varepsilon_{\mathrm{PS}-\mathrm{PB}}$ is then fit to SANS data for the binary system, and some degree of inherent stiffness (chain semiflexibility) is ascribed to polymer chains to model the presence of steric interactions. The heterocontant energies $\varepsilon_{\mathrm{PS}-\mathrm{PB}}$ likewise emerge as virtually independent of $\mathrm{dPB}$ microstructure, and the $\mathrm{dPB}$ chain stiffness is found to increase, as expected, 
with the degree of branching (1,2 addition units). The lack of variation of energies with microstructure and the increased dPB stiffness with higher degrees of branching indicate that the LCT provides a reasonably faithful molecular representation of the systems. However, the dPB/PS systems are far from ideal for a thorough quantitative test of the capabilities of the LCT for several reasons as follows: The very slightly miscibility of PB and PS requires the use of samples with rather low molecular weights (see Table I), and, consequenty, the chemically distinct end groups have a nonnegligible influence ${ }^{13}$ on the system thermodynamics. Since modeling the presence of chemically distinct end groups would require introducing additional interaction energies, we ignore the presence of these different end groups, thereby naturally incurring some quantitative errors in the fitting process. Moreover, the three dPB samples are random copolymers, but we treat them, for simplicity, as pure homopolymers, neglecting the $7 \%$ of 1,2 units in the nominally $1,4-$ $\mathrm{dPB}$ and the $9 \%$ of 1,4 units in the nominally $1,2-\mathrm{dPB}$ and describing the 50-50 1,2/1,4 random copolymer as a purely alternating homopolymer. Thus, our goal here lies in a semiquantitative analysis of the various molecular factors influencing the pressure and microstructure dependence of the enthalpic and entropic portions of the experimental interaction parameter $\Gamma=-\Gamma_{\sigma}+\Gamma_{h} / T$.

The theoretical representation of the equation of state data (see Fig. 3) is quite reasonable considering the modeling limitations noted above and the fact that only two parameters are used for each pure component whereas all other theories of melt $p V T$ data require at least three adjustable parameters. When applied to a dPB/PS "homopolymer" blend, the theory employs as additional adjustable parameters the heterocontact interaction energy $\epsilon_{\mathrm{PS}-\mathrm{PB}}$ and the two bending energies $\epsilon_{\text {bend }}^{(\mathrm{PB})}$ and $\epsilon_{\text {bend }}^{(\mathrm{PS})}$. The agreement between theory and experiments (see Figs. 6-8) is quite reasonable in the sense that the theory properly describes the pressure dependence of $\Gamma$. The computed values of $\Gamma_{\sigma}$ and $\Gamma_{h}$ deviate from the experimental slopes $\Gamma_{h}$ and intercepts $\Gamma_{\sigma}$ in the temperature dependence $\Gamma=-\Gamma_{\sigma}+\Gamma_{h} / T$, but the deviation occurs in a form commonly found ${ }^{10,11,23,24}$ when $\Gamma_{\sigma}$ is moderately large and positive and when $\Gamma_{h}$ is also positive, indicating a general limitation of the LCT or, perhaps, problems associated with the simplifying assumptions invoked as discussed above. (The experimental $\Gamma_{\sigma}$ is positive for all three blend samples, whereas the theoretical LCT $\Gamma_{\sigma}$ is negative.) $\mathrm{Nu}-$ merous applications of the binary homopolymer blend
$\mathrm{LCT}^{10,11,23,24}$ indicate, on the other hand, that the theory successfully describes the experimental interaction parameter $\Gamma$ when its temperature-independent portion $\Gamma_{\sigma}$ is negative and rather large. A negative $\Gamma_{\sigma}^{\mathrm{LCT}}$ also results from the infinite pressure, high molecular weight, fully flexible chain limit of the LCT [see Eq. (16)].

\section{ACKNOWLEDGMENTS}

The authors gratefully thank T. Pakula and S. Klein from the Max Planck Institute for Polymer Research in Mainz for the opportunity they afforded for measuring the pVT equation of state. This research is supported, in part, from Grant No. PKZ D/97/16489 from DAAD and from Grant No. DAAG55-97-1-0162 from ARO.

${ }^{1}$ P. G. DeGennes, Scaling Concepts in Polymer Physics (Cornell University, Ithaca, NY, 1979).

${ }^{2}$ K. Binder, Adv. Polym. Sci. 112, 181 (1994).

${ }^{3}$ H. Frielinghaus, D. Schwahn, and L. Willner, Macromolecules (in press).

${ }^{4}$ D. Schwahn, G. Meier, K. Mortensen, and S. Jansen, J. Phys. II 4, 837 (1994).

${ }^{5}$ G. Meier, D. Schwahn, K. Mortensen, and S. Jansen, Europhys. Lett. 22, 577 (1993).

${ }^{6}$ J. Dudowicz, M. S. Freed, and K. F. Freed, Macromolecules 24, 5076 (1991); Macromolecules 24, 5096 (1991); Macromolecules 24, 5112 (1991).

${ }^{7}$ S. Janßen, D. Schwahn, and T. Springer, Phys. Rev. Lett. 68, 3180 (1992).

${ }^{8}$ P. J. Flory, J. Chem. Phys. 9, 660 (1941); M. L. Huggins, ibid. 9, 440 (1941).

${ }^{9}$ S. Janßen, D. Schwahn, T. Springer, K. Mortensen, and H. Hasegawa, Physica B 213, 691 (1995).

${ }^{10}$ J. Dudowicz and K. F. Freed, Macromolecules 28, 6625 (1995).

${ }^{11}$ K. F. Freed and J. Dudowicz, Macromolecules 31, 6681 (1998).

${ }^{12}$ J. Dudowicz and K. F. Freed, Macromolecules 33, 3467 (2000).

${ }^{13}$ H. Frielinghaus, W. B. Pedersen, P. S. Larsen, K. Almdal, and K. Mortensen, Macromolecules (in press).

${ }^{14}$ K. W. Foreman and K. F. Freed, J. Chem. Phys. 107, 4688 (1997).

${ }^{15}$ M. Y. Belyakov and S. B. Kiselev, Physica A 190, 75 (1992).

${ }^{16}$ J. Dudowicz, M. Lifschitz, K. F. Freed, and J. F. Douglas, J. Chem. Phys. 99, 4804 (1993).

${ }^{17}$ M. A. Anisimov, S. B. Kiselev, J. V. Sengers, and S. Tang, Physica A 188, 487 (1992).

${ }^{18}$ M. Lifschitz, J. Dudowicz, and K. F. Freed, J. Chem. Phys. 100, 3957 (1994).

${ }^{19}$ K. W. Foreman and K. F. Freed, J. Chem. Phys. 106, 7422 (1997).

${ }^{20}$ K. W. Foreman, K. F. Freed, and I. M. Ngola, J. Chem. Phys. 107, 4688 (1997).

${ }^{21}$ K. W. Foreman and K. F. Freed, Adv. Chem. Phys. 103, 335 (1998).

${ }^{22}$ J. Higgins and H. Benoît, Polymers and Neutron Scattering (Clarendon, Oxford, U.K., 1994).

${ }^{23}$ C. Delfolie, L. C. Dickinson, K. F. Freed, J. Dudowicz, and W. J. MacKnight, Macromolecules 32, 7781 (1999).

${ }^{24}$ J. Dudowicz and K. F. Freed, Macromolecules 33, 3467 (2000); Pol. J. Chem. (in press). 